\title{
Investigation on Dynamics of Mechanical System with Clearance Joint
}

\author{
Zheng-Feng Bai ${ }^{*}, 1$, Bin-jiu Yang ${ }^{1}$ and Yi Sun ${ }^{2}$ \\ ${ }^{1}$ Department of Mechanical Engineering, Harbin Institute of Technology at Weihai, Weihai 264209, P.R. China \\ ${ }^{2}$ Department of Astronautic Science and Mechanics, Harbin Institute of Technology, Harbin 150001, P.R. China
}

\begin{abstract}
In this work, the dynamic responses and vibration characteristics of mechanical system with revolute clearance joints are investigated numerically. Considering clearance in joint, the intra-joint contact model that is generated at clearance joints is established using a nonlinear continuous contact force model and the friction effect is considered by using a modified Coulomb friction model. A well-known slider-crank mechanism with a revolute clearance joint is utilized to perform the investigation. The effects of clearance on dynamics of mechanical system are analyzed by timedomain responses and frequency domain analysis. The investigation results show that the dynamic responses of mechanical system with clearance are obviously vibration and the amplitude increases from the mechanism without clearance. The results also indicate that the behavior of mechanism with clearance is nonlinear.
\end{abstract}

Keywords: Clearance joint, mechanical system, numerical simulation, vibration characteristics.

\section{INTRODUCTION}

Clearances in mechanism are unavoidable due to assemblage, manufacturing errors and wear. Moreover, clearance occurs in each active joint with the movement of mechanism. The movement of the real mechanisms is deflected from the ideal mechanism and the motion accuracy is decreased due to joint clearances. It is known as a source of impact forces and these forces not only create increasing vibration amplitude, but also reduce system reliability, stability, life, and precision [1-4]. These clearances modify the dynamic responses of the system, justify the deviations between the numerical predictions and experimental measurements and eventually lead to significant vibrations of the real mechanism [5-11].

Over the last few decades, effects of clearance on dynamic responses of mechanisms using theoretical and experimental approaches have been studied by many researchers. Stoenescu and Marghitu (2003) [12] investigated the dynamic response of a planar, rigid-link mechanism with a sliding joint clearance and the response of the system with clearance was chaotic at relatively high crank speeds and low values of the coefficient of restitution. Zhao and Bai (2011) [13] studied the dynamics of a space robot manipulator with one joint clearance. The nonlinear equivalent spring-damper model is established for the contact model in joint clearance. Also, the friction effect is considered using the Coulomb friction model. Flores et al. (2004) [14] presented dynamic analysis of planar multi-body systems with revolute joint clearances, including dry contact and lubricant effect. Recently, Flores (2010) [15] presented a parametric study on the dynamic response of planar multi-body systems with multiple clearance joints. An elementary slider-crank

*Address correspondence to this author at the No.2, Wen-hua West Road, Weihai, Shandong. Postcard: 264209, China; Tel: +86-631-5687026; E-mail: baizhengfeng@126.com mechanism was used to perform the parametric study. The main functional parameters were used for this study, namely, the diametric clearance size, the input crank speed and the number of joints modeled as clearance joints. Erkaya (2010) [16] presented an experimental study, in which a slider-crank mechanism with clearance joints was discussed. Rhee and Akay (1996) [17] investigated the dynamic response of a revolute joint with clearance. A four-bar mechanism was implemented as an example to be used to model the motion of a rocker arm pin at the ground connection. Bai and Zhao (2012) [18] proposed a nonlinear hybrid contact force model, which was applied in dynamics analysis of mechanical systems with revolute clearance joint. The dynamics responses obtained with numerical models are compared with that of experimental results. More recently, Koshy [19] presented a computational and experimental study on the contact forces developed in revolute clearance joints. For this purpose, a well-known slidercrank mechanism with a revolute clearance joint between the connecting rod and slider is analyzed.

All the researches indicate that clearance has significant effects on dynamic response of mechanism system. However, previous researches mainly focused on the time domain dynamic responses and less focused on frequency domain analysis of mechanical system with clearance. Thus, the dynamics of mechanical system with revolute clearance joints are analyzed by time domain responses and frequency domain analysis utilizing Fast Fourier Translation (FFT). The effects of clearance on dynamics and vibration characteristics of mechanical system are discussed. The planar slider-crank mechanism with revolute clearance joint is used as numerical example to perform the investigation.

\section{MODEL FOR REVOLUTE CLEARANCE JOINT}

\subsection{Definition of Clearance}

In general, a clearance joint can be included in a mechanical system much like a revolute joint. The classical 
approach, known as zero-clearance approach, assumes that the connecting points of two bodies linked by a revolute joint are coincident. Then the clearance produced in a joint separates these two points. Fig. (1) depicts a revolute joint with clearance. The difference in radii between the bearing and journal defines the size of the radial clearance. Although, a revolute joint with clearance does not impart any degree of freedom constraint from the mechanical system like the ideal joint, it imposes some kinematic restrictions, limiting the journal to move within the bearing. Thus, when clearance is present in a revolute joint, the two kinematic constraints are removed and two degrees of freedom are introduced instead. The dynamics of the joint are then controlled by forces working on the journal and bearing. Thus, whilst a perfect revolute joint in a mechanical system imposes kinematic constraints, a revolute clearance joint leads to forced constraints. When contact exists between the journal and bearing, a contact force is applied perpendicular to the plane of collision. Therefore, the motion of mechanical system with clearance always includes contact-impact process, which needs accurate definition of the contact-impact process in the joint clearance.

The radial clearance is defined as follows,

$$
c=R_{B}-R_{J}
$$

where $R_{B}$ and $R_{J}$ are the radii of bearing and journal, respectively.

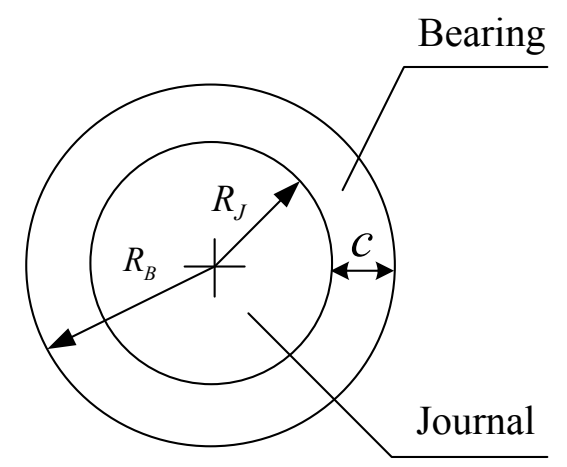

Fig. (1). Schematic of revolute joints with clearance.

\subsection{Mathematic Model of Joint with Clearance}

As is shown in Fig. (2), $O_{i}$ and $O_{j}$ define the centers of bearing and journal, respectively, $\boldsymbol{r}_{i}^{o}$ and $\boldsymbol{r}_{j}^{o}$ are the vectors denoting the positions of bearing and journal in the global inertia coordinate, respectively. So in Fig. (2), the clearance vector is represented as equation (2):

$$
\boldsymbol{e}_{i j}=\boldsymbol{r}_{j}^{o}-\boldsymbol{r}_{i}^{o}
$$

where, $\boldsymbol{e}_{i j}$ represents the eccentric vector of journal relative to bearing. So the eccentricity of clearance vector can be represented as follows:

$e_{i j}=\sqrt{e_{x}^{2}+e_{y}^{2}}$

The unit normal vector of bearing and journal at the point of their contact is represented as equation (4): $\boldsymbol{n}=\boldsymbol{e}_{i j} / e_{i j}$

Fig. (3) describes the collision between bearing and journal. $\boldsymbol{r}_{i}^{Q}$ and $\boldsymbol{r}_{j}^{Q}$ are the positional vectors of the bearing and journal, respectively, which are described in global coordinate frame. The contact deformation caused by collision between bearing and journal can be represented as equation (5):

$\delta=e_{i j}-c$

where, $c$ represents the clearance and it is a constant.

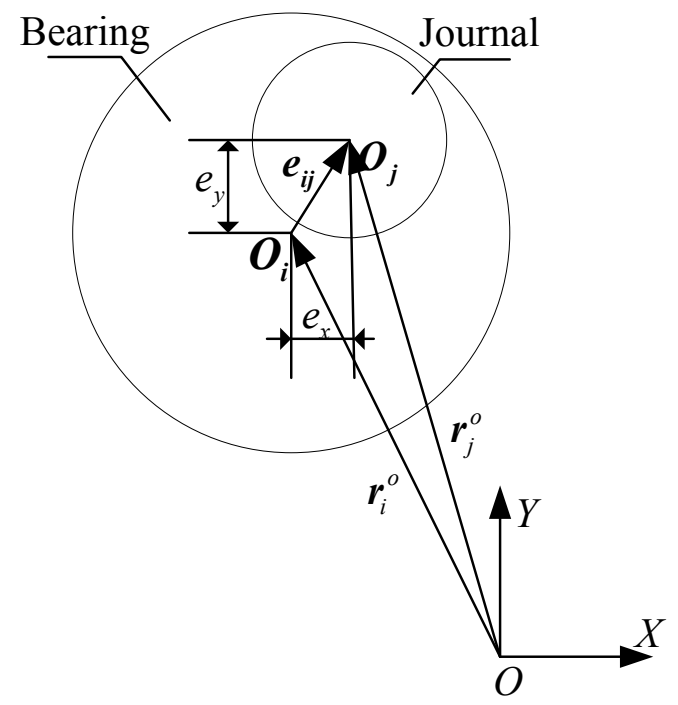

Fig. (2). Clearance model in revolute joint.

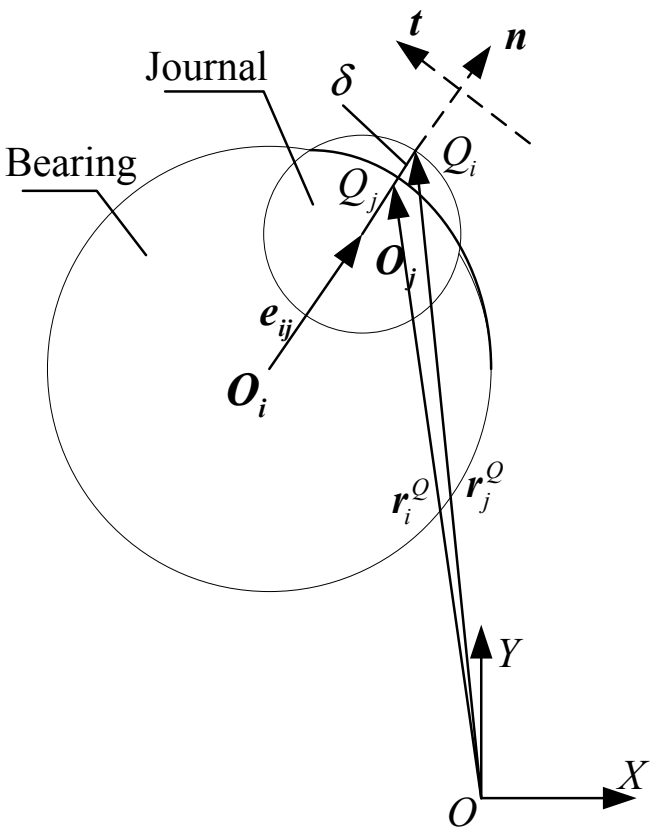

Fig. (3). Revolute clearance joint with contact.

Thus, $\delta$ can be used to find whether the bearing and journal have contacted. The kinematic contact condition between the bearing and journal can be given by equation (6): 
$\delta=\sqrt{e_{x}^{2}+e_{y}^{2}}-c \geq 0$

Since it is important to estimate the loss of energy in the course of contact between bearing and journal, so it is necessary to calculate the relative speed between their surfaces. When we project $\delta$ to the facet of contact, we can get the normal speed and tangential speed of the potential points of contact between the bearing and journal, as shown in equation (7):

$\boldsymbol{v}_{n}=(\dot{\delta})^{T} \boldsymbol{n}$

$\boldsymbol{v}_{t}=(\dot{\delta})^{T} \boldsymbol{t}$

where, the unit tangential vector $t$ can be achieved by reversing the unit normal vector $\boldsymbol{n}$ for $90^{\circ}$

\section{CONTACT FORCE MODEL IN CLEARANCE JOINT}

Contact and impact are the typical phenomena of multibody mechanical system with joint clearance. The contact-impact model of multibody system with clearance is mainly focused on the discrete analysis method and continuous contact analysis method. The former assumes that the contact-impact is very short and did not change the overall configuration of the objects. Then, the contact-impact process is divided into two stages, i.e. before and after impact. And relative sliding, viscous stagnation and reverse movement will occur between two objects after the impact. The latter assumes that interaction forces between the impact objects are continuous in the entire contact-impact process. This approach tallies with real contact-impact behavior of objects. The continuous contact force model is widely used for contact-impact analysis of mechanism with clearance. Bai and Zhao (2013) [4] proposed a nonlinear continuous contact force model in revolute clearance joint, which is a hybrid model of the Lankarani-Nikravesh model and the improved Winkler elastic foundation model. This contact force model considers the effect of damping and describes the energy loss in the contact process. In present work, this nonlinear continuous contact force model is used for contact modeling of journal and bearing in revolute clearance joint with clearance for solar panel.

The expression of the nonlinear continuous contact force model is expressed as:

$$
F_{n}=K_{n} \delta^{n}+D_{\text {mod }} \dot{\delta}
$$

where, the elastic deformation force is represented by the first item of the right side of equation (8) and the energy loss is represented by the second item. $\delta$ is the deformation, and $\dot{\delta}$ is the relative deformation velocity. $K_{n}$ is the nonlinear stiffness coefficient of the impact body and $D_{\bmod }$ is the modified damping coefficient.

The nonlinear stiffness coefficient, $K_{n}$, is obtained from the following equation:
$K_{n}=\frac{1}{8} \pi E^{*} \sqrt{\frac{2 \delta\left(3\left(R_{B}-R_{J}\right)+2 \delta\right)^{2}}{\left(R_{B}-R_{J}+\delta\right)^{3}}}$

where, $R_{B}$ and $R_{J}$ are radii of the bearing and journal, respectively. $E^{*}$ is compound elastic modulus and the expression is represented as:

$\frac{1}{E^{*}}=\frac{1-v_{i}^{2}}{E_{i}}+\frac{1-v_{j}^{2}}{E_{j}}$

where, $v$ and $E$ are Poisson ratio and Young modulus, respectively.

The nonlinear stiffness coefficient, $K_{n}$, is related to the material property, geometry property, clearance size and deformation of contact bodies, it varies with $\delta$, and is not constant during the contact process.

Coefficient, $D_{\text {mod }}$, in equation (8) is a modified damping coefficient, and the expression of $D_{\bmod }$ is shown as equation (11):

$D_{\text {mod }}=\frac{3 K_{n}\left(1-c_{e}^{2}\right) e^{2\left(1-c_{e}\right)} \delta^{n}}{4 \dot{\delta}^{(-)}}$

where, $c_{e}$ is coefficient of restitution and $\dot{\delta}^{(-)}$is initial relative velocity of the impact point.

\section{FRICTION FORCE MODEL IN REVOLUTE CLEARANCE JOINT}

It is known that the Coulomb law of sliding friction can represent the most fundamental and simplest model of friction between dry contacting surfaces. The tangential contact characteristic of clearance is represented using tangential friction force model. Thus, in this paper, the friction effects in joints are considered as dry friction and a modified Coulomb friction model is used to represent the friction response between the journal and bearing. Friction coefficient, which is not a constant, is introduced in the modified Coulomb friction model. Friction coefficient is a function of tangential sliding velocity, which can represent the friction response in impact and contact process as well as the viscous and micro-slip phenomenon in relative lowvelocity case more accurately. And also, the modified Coulomb friction model can avoid the case of abrupt change of friction in numerical calculation as the change of velocity direction.

The expression of tangential friction force is shown as equation (12):

$F_{t}=-\mu\left(\boldsymbol{v}_{t}\right) F_{n} \frac{\boldsymbol{v}_{t}}{\left|\boldsymbol{v}_{t}\right|}$

where, friction coefficient $\mu\left(\boldsymbol{v}_{t}\right)$ is a function of tangential sliding velocity, which can be expressed as equation (13): 


$$
\mu\left(v_{t}\right)=\left\{\begin{array}{cl}
-\mu_{d} \operatorname{sign}\left(v_{t}\right) & \text { for } \quad\left|v_{t}\right|>v_{d} \\
-\left\{\mu_{d}+\left(\mu_{s}-\mu_{d}\right)\left(\frac{\left|v_{t}\right|-v_{s}}{v_{d}-v_{s}}\right)^{2}\left[3-2\left(\frac{\left|v_{t}\right|-v_{s}}{v_{d}-v_{s}}\right)\right]\right\} \operatorname{sign}\left(v_{t}\right) & \text { for } \quad v_{s} \leq\left|v_{t}\right| \leq v_{d} \\
\mu_{s}-2 \mu_{s}\left(\frac{v_{t}+v_{s}}{2 v_{s}}\right)^{2}\left(3-\frac{v_{t}+v_{s}}{v_{s}}\right) & \text { for } \quad\left|v_{t}\right|<v_{s}
\end{array}\right.
$$

where, $v_{t}$ is a relative sliding velocity in the collision point of journal and bearings, and is the velocity component in tangential direction. $\mu_{d}$ is the dynamic friction coefficient. $\mu_{s}$ is the static friction coefficient. $v_{s}$ is the critical velocity of static friction. $v_{d}$ is the critical velocity of the maximum dynamic friction.

\section{DEMONSTRATIVE APPLICATION EXAMPLE}

\subsection{Slider-Crank Mechanism with Revolute Clearance Joint}

In this section, the dynamic responses and vibration characteristics of mechanical systems with revolute clearance joint are investigated numerically. The planar slider-crank mechanism with revolute clearance joint is used as numerical example to demonstrate the investigation. The effects of clearance on dynamics of multibody mechanical system with revolute clearance joints are presented by time domain responses and frequency domain analysis utilizing Fast Fourier Translation (FFT).

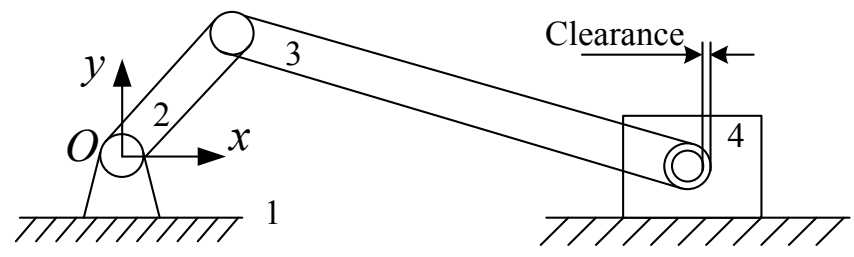

Fig. (4). Slider-crank mechanism with a clearance joint.

Fig. (4) depicts the kinematic configuration of the slidercrank mechanism, which consists of four bodies, including ground, two ideal revolute joints, and one ideal translational joint. A revolute clearance joint exists between the connecting rod and slider. The body numbers and their corresponding coordinate systems are shown in Fig. (4). In order to keep the analysis simple and to illustrate the dynamic responses of clearance joint, all the bodies are considered to be rigid. The length and inertia properties of the slider-crank mechanism components are listed in Table $\mathbf{1}$ and the parameters used in the dynamic simulations are given in Table 2.

In the dynamic simulation the crank is the driving body which rotates with a constant angular velocity equal to 200r/min. The initial configuration corresponds to crank and connecting rod collinear, and the position and velocity journal centers are taken to be zero. Initially, the journal and bearing centers are coincidental. Note that the results are plotted against those obtained for ideal joint, being reported for the two full crank rotations after a steady-state has been reached.

Table 1. Geometric and inertia properties of the crank-slider mechanism.

\begin{tabular}{|c|c|c|c|}
\hline Body & Length (m) & Mass (kg) & Moment of Inertia $\left(\mathbf{k g m}^{2}\right)$ \\
\hline \hline Crank & 0.05 & 17.900 & 0.460327 \\
\hline Connection rod & 0.3 & 1.130 & 0.015300 \\
\hline Sliding block & - & 1.013 & 0.000772 \\
\hline
\end{tabular}

Table 2. Simulation parameters for the experimental slidercrank mechanism.

\begin{tabular}{|l|c|}
\hline Restitution Coefficient & 0.46 \\
\hline Dynamic friction coefficient & 0.01 \\
\hline Young's modulus & $207 \mathrm{GPa}$ \\
\hline Poisson's ratio & 0.3 \\
\hline
\end{tabular}

\subsection{Numerical Results and Discussion}

In the dynamic simulation, the crank is the driving body which rotates with a constant angular velocity equal to 200r/min. The contact force model in clearance joint uses the nonlinear contact force mode given by Equation (8). The values of the clearance size are considered as $1 \mathrm{~mm}, 0.5 \mathrm{~mm}$, $0.25 \mathrm{~mm}$ and $0.1 \mathrm{~mm}$.

Fig. (5) presents the effects of clearance on acceleration of slider. It can be found that the effects of clearance on dynamic behavior of mechanism cannot be ignored. The acceleration of slider with clearance is obviously shaking and the amplitude increases from the mechanism without clearance, which indicates that the existence of clearance will lead to oscillation mechanism. From Fig. (5), it also can be found that when the clearance size increases, the dynamics responses of mechanism are changed obviously, which is represented by vibration with higher peaks. It indicates that the higher size of clearance will lead to the more obvious vibration and higher peaks of acceleration.

Further, the vibration characteristics of mechanical systems with clearance are discussed. A Fast Fourier Transformation is performed to analyze the frequency spectrum of the vibration characteristics. Fig. (6) shows the outputs from the FFT analysis. 
(a)

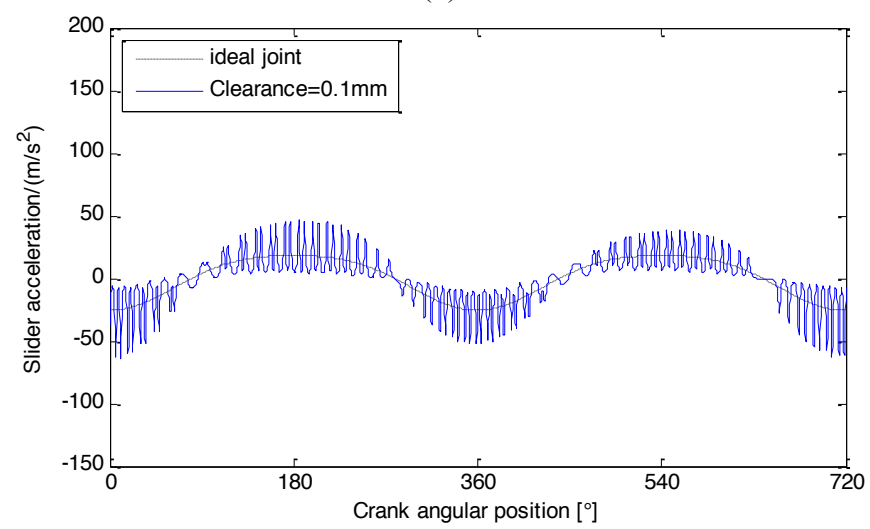

(b)

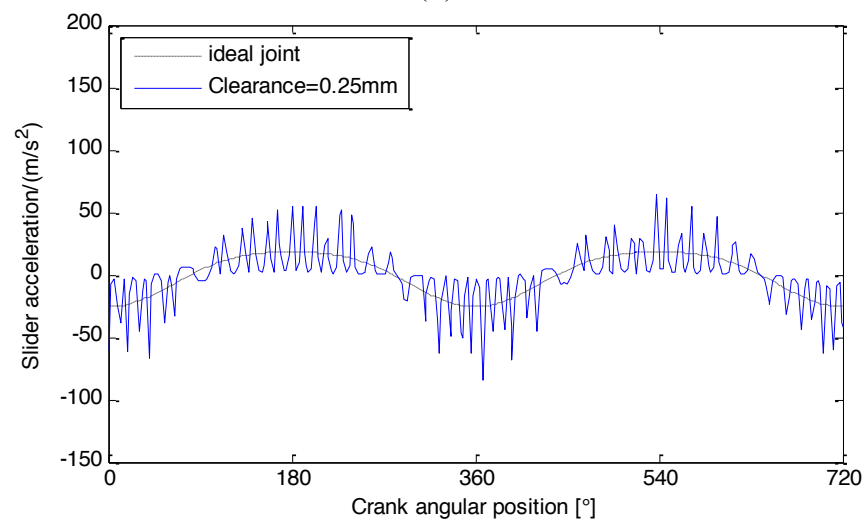

(c)

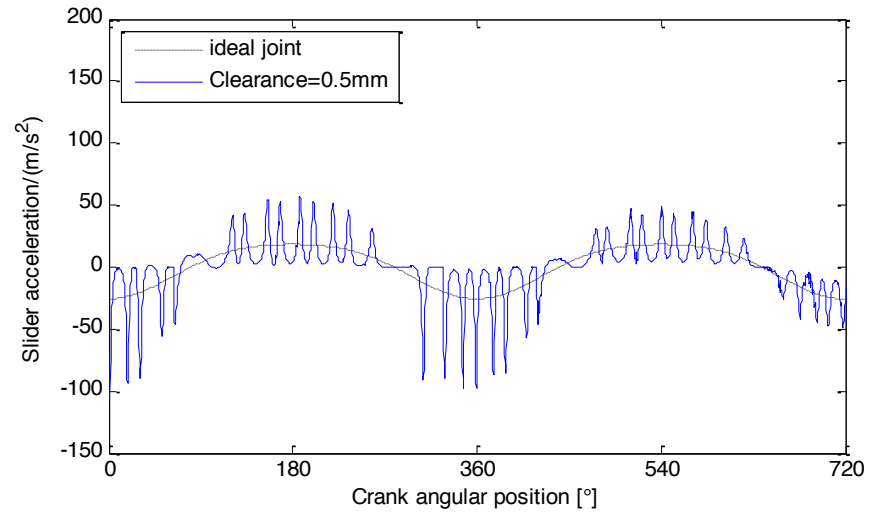

(d)

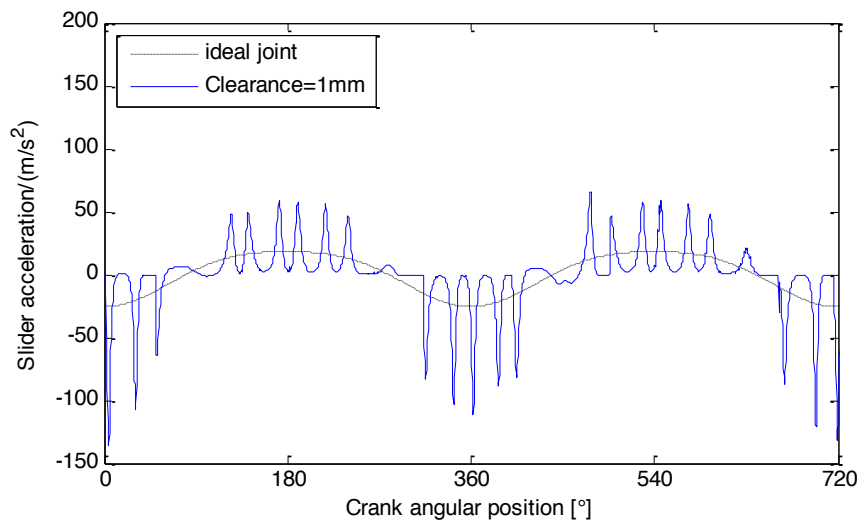

Fig. (5). Numerical slider acceleration for different clearance sizes: (a) $\mathrm{c}=0.1 \mathrm{~mm},(\mathbf{b}) \mathrm{c}=0.25 \mathrm{~mm},(\mathbf{c}) \mathrm{c}=0.5 \mathrm{~mm},(\mathbf{d}) \mathrm{c}=1.0 \mathrm{~mm}$. (a)

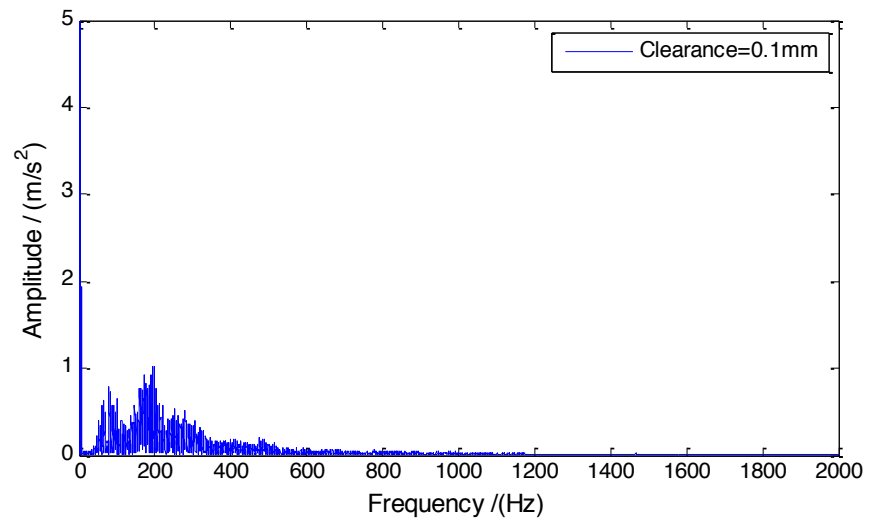

(b)

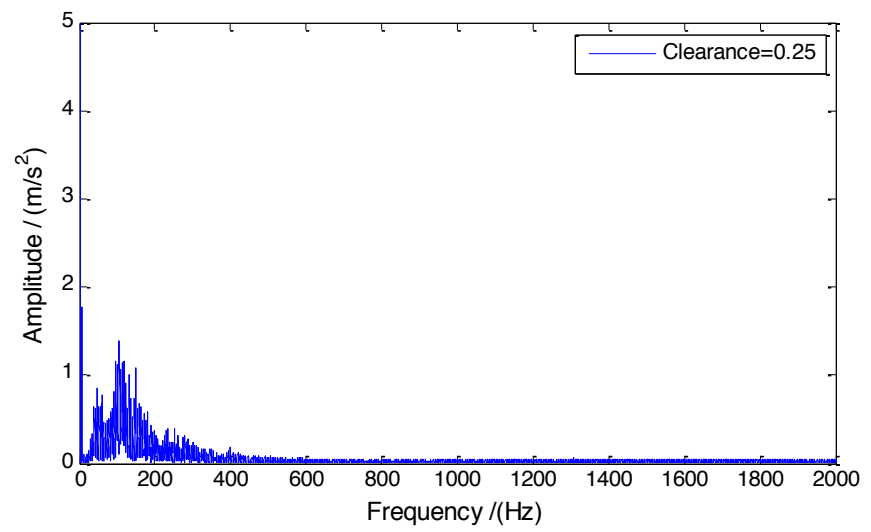

(c)

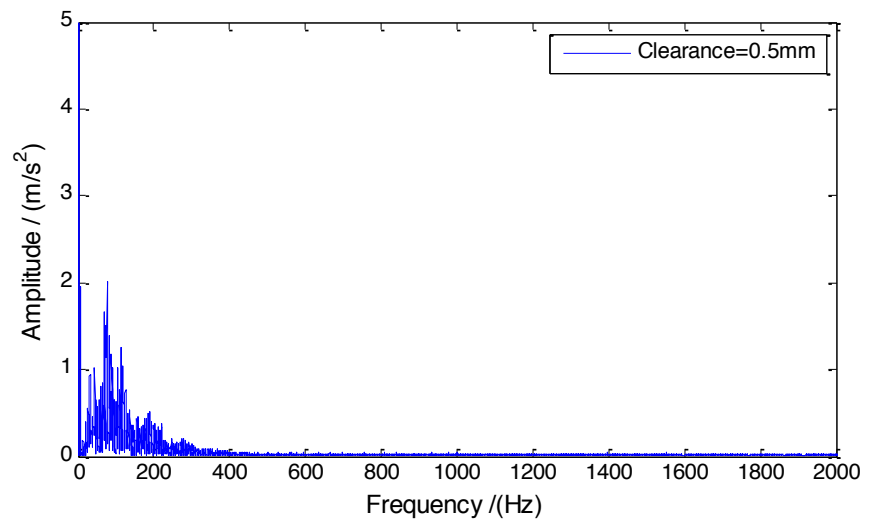

(d)

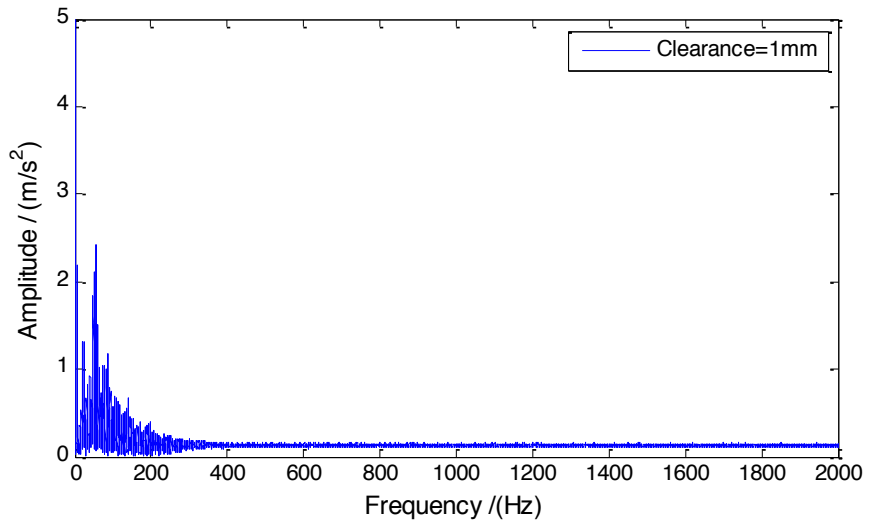

Fig. (6). FFT analysis of acceleration with different clearance sizes: (a) $\mathrm{c}=0.1 \mathrm{~mm}$, , (b) $\mathrm{c}=0.25 \mathrm{~mm}$, (c) $\mathrm{c}=0.5 \mathrm{~mm}$, (d) $\mathrm{c}=1.0 \mathrm{~mm}$. 
As shown in Fig. (6), the peaks of vibration amplitude increase as the clearance size increases. For each clearance size, the highest peak of amplitude is appeared near $3.32 \mathrm{~Hz}$, which is the dominating frequency of the periodic crank rotation. However, as shown in Fig. (6a), when clearance size is $0.1 \mathrm{~mm}$, the larger amplitude of the vibration response is in the frequency range below $400 \mathrm{~Hz}$ and there is another peak of amplitude at $196.8 \mathrm{~Hz}$. However, when the clearance sizes are $0.25 \mathrm{~mm}$, $0.5 \mathrm{~mm}$ and $1 \mathrm{~mm}$, as shown in Fig. (6b-d), the larger amplitude of the vibration response are in the frequency range below $300 \mathrm{~Hz}, 200 \mathrm{~Hz}$ and $100 \mathrm{~Hz}$, respectively. Besides, there are other peaks of amplitude at $106.7 \mathrm{~Hz}, 75.84 \mathrm{~Hz}$ and $55.51 \mathrm{~Hz}$, when clearance sizes are $0.25 \mathrm{~mm}, 0.5 \mathrm{~mm}$ and $1 \mathrm{~mm}$, respectively. Therefore, it indicates that the bigger size of clearance will lead to the lower frequency of contact in clearance joint, which is due to the bearing and journal being in contact for a longer period with each other when the clearance is bigger. It indicates that bigger clearance size results in less contact between the journal and the bearing, and the contact period is increased.

\section{CONCLUSION}

The dynamic responses and vibration characteristics of multibody mechanical system with revolute clearance joint are investigated using a computational method. The contact models that are generated at clearance joints is established using a nonlinear continuous contact force model and the friction effect is considered by using a modified Coulomb friction model. A well-known slider-crank mechanism with a revolute clearance joint is utilized to perform the investigation. The effects of clearance on dynamics of mechanical system are analyzed by time domain responses and frequency domain analysis. A Fast Fourier Transformation is performed to analyze the frequency spectrum of the vibration characteristics.

The effects of clearance on the dynamic responses of mechanism cannot be ignored, and the existence of clearance causes acceleration at a very high frequency vibration and decrease of the motion stability. The behavior of mechanism with clearance is nonlinear. In addition, when the clearance size increases, the dynamics responses of mechanism are changed obviously, and are represented by vibration with higher peaks. It also shows that the larger size of the clearance will lead to the more obvious vibration and higher peaks of acceleration. However, the larger size of clearance will lead to the lower frequency of contact in clearance joint. Therefore, clearance size will significantly affect the dynamic responses and vibration characteristics of the system.

A future and natural extension of our work is to analyze the dynamics of multibody system with clearance joint including link flexibility, as well as the experimental study, which will give a more accurate response of mechanism caused by joint clearance.

\section{CONFLICT OF INTEREST}

The authors confirm that this article content has no conflict of interest.

\section{ACKNOWLEDGEMENTS}

This research was supported by the National Natural Science Foundation of China (51305093), the Natural Science Foundation of Shandong Province (ZR2013EEQ004) and the National Key Basic Research Program of China (2013CB733000). Project supported by the China Postdoctoral Science Foundation funded project (2012M520723; 2014T70317) is also gratefully acknowledged.

\section{REFERENCES}

[1] M. Machado, Pe. Moreira, P. Flores, and H.M. Lankarani, "Compliant contact force models in multibody dynamics Evolution of the Hertz contact theory", Mech. Mach. Theory, vol. 53, pp. 99-121, 2012

[2] Z.F. Bai, Y. Zhao, and X.G. Wang, "Wear analysis of revolute joints with clearance in multibody systems", Sci. China Phys. Mech. vol. 56, pp. 1581-1590, 2013

[3] C.S. Liu, K. Zhang, and R. Yang, "The FEM analysis and approximate model for cylindrical joints with clearances", Mech. Mach. Theory, vol. 42, pp.183-197, 2007

[4] Z.F. Bai, and Y. Zhao, "A hybrid contact force model of revolute joint with clearance for planar mechanical systems", Int. J. Nonlin. Mech., vol. 48, pp.15-36, 2013.

[5] S. Erkaya, and I. Uzmay, "A neural-genetic (NN-GA) approach for optimizing mechanisms having joints with clearance", Multibody Syst. Dyn., vol. 20, pp. 69-83, 2008

[6] Z.F. Bai, Y. Zhao, and J. Chen, "Dynamics analysis of planar mechanical system considering revolute clearance joint wear", Tribol. Int., vol.64, pp. 85-95, 2013

[7] I. Khemili, and L. Romdhane, "Dynamic analysis of a flexible slidercrank mechanism with clearance", Eur. J. Mech. A-Solid, vol. 27, pp. 882-898, 2008.

[8] Q. Tian, Y.Q. Zhang, L.P. Chen, and P. Flores, "Dynamics of spatial flexible multibody systems with clearance and lubricated spherical joints", Comput. Struct., vol. 87, pp. 913-929, 2009.

[9] S. Erkaya, "Investigation of joint clearance effects on welding robot manipulator", Robot. Cim-Int Manuf., vol. 28, pp.449-457, 2012

[10] O.A. Bauchau, and J. Rodriguez, "Modeling of joints with clearance in flexible multi-body system", Int. J. Solids Struct., vol. 34, pp.41-63, 2002.

[11] A.L. Schwab, J.P. Meijaard, and P. Meijers, "A Comparison of revolute joint clearance model in the dynamic analysis of rigid and elastic mechanical systems", Mech. Mach. Theory, vol. 37, pp. 895-913, 2002

[12] E.D. Stoenecu, and D.B. Marghitu, "Dynamic analysis of a planar rigidlink mechanism with rotating slider joint and clearance", J. Sound Vib., vol. 266, pp.394-404, 2003.

[13] Y. Zhao, and Z.F. Bai, "Dynamics analysis of space robot manipulator with joint clearance," Acta Astronaut., vol. 68, pp.1147-1155, 2011

[14] P. Flores, J. Ambrósio, and J.P. Claro, "Dynamic analysis for planar multibody mechanical systems with lubricated joints", Multibody Syst. Dyn., vol. 12, pp. 47-74, 2004

[15] P. Flores, "A parametric study on the dynamic response of planar multibody systems with multiple clearance joints", Nonlinear Dyn., vol. 61, pp. 633-653, 2010.

[16] S. Erkaya, and I. Uzmay, "Experimental investigation of joint clearance effects on the dynamics of a slider-crank mechanism", Multibody System Dynamics, vol. 24, 81-102, 2010

[17] J. Rhee, A. Akay, A., Dynamic response of a revolute joint with clearance, Multibody Syst. Dyn., vol. 31, pp.121-134, 1996.

[18] Z.F. Bai, and Y. Zhao, "Dynamic behaviour analysis of planar mechanical systems with clearance in revolute joints using a new hybrid contact force model", Int. J. Mech. Sci., vol. 54, pp. 190-205, 2012.

[19] C.S. Koshy, P. Flores, and H.M. Lankarani, "Study of the effect of contact force model on the dynamic response of mechanical systems with dry clearance joints-computational and experimental approaches", Nonlinear Dyn., vol. 73, pp. 325-338, 2013. 